\title{
966.
}

\section{NOTE ON A MEMOIR IN SMITH'S COLLECTED PAPERS.}

[From the Bulletin of the American Mathematical Society, Ser. 2, vol. I. (1895), pp. 94-96.]

Among the most noticeable papers in the Collected Mathematical Papers of H. J. S. Smith we have the hitherto unpublished "Memoir on the Theta and Omega Functions," XLIII. (vol. II. pp. 415-623), written in connexion with Dr Glaisher's Tables of the Theta-Functions and originally intended as an Introduction thereto. It appears that in 1873 or 1874 Dr Glaisher asked him, as a member of the British Association committee for the calculation of the Tables, whether he would contribute an Introduction. His reply was that he did not see his way to writing anything appropriate to the tables themselves, but that he "could say something with respect to the constants at the head of the pages." These constants were $K, K^{\prime}, E, J, J^{\prime}$, \&c., the numerical values whereof were given for every minute of the modular angle. The memoir grew in extent, and it was finally decided that it should follow these yet unpublished tables with the before-mentioned title, "Memoir on the Theta and Omega Functions," but fortunately it has at length appeared in the Collected Papers as above.

In explanation of the title and scope of the memoir, it will be remembered that the Theta-Functions are functions of two arguments, $x$ and $q$; so that giving to $x$ the value zero or any numerical value, or any value depending on that of $q$, we obtain a series of functions containing the single argument $q$, or writing as usual $q=e^{i \pi \omega}$, say the single argument $\omega$; and in the memoir, the attention is directed chiefly but not exclusively to these functions of a single argument which are termed Omega Functions. The functions chiefly considered under this designation are Hermite's functions $\phi \omega, \psi \omega, \chi \omega$, which represent the values of $\sqrt{k}, \sqrt[4]{k^{\prime}}$, and $\sqrt[12]{k k^{\prime}}$ considered as functions of $q=e^{i \pi \omega}$. To fix the ideas, it may be mentioned that the actual values (in one of their very numerous forms) are

$$
\begin{aligned}
& \phi \omega=\sqrt{2} q_{\frac{1}{8}} \prod_{1}^{\infty} \frac{1+q^{2 m}}{1+q^{2 m-1}}, \\
& \psi \omega=\prod_{1}^{\infty} \frac{1-q^{2 m-1}}{1+q^{2 m-1}}, \\
& \chi \omega=\sqrt[6]{2} q_{\frac{1}{24}} \prod_{1}^{\infty} \frac{1}{1+q^{2 m-1}},
\end{aligned}
$$


each of them a one-valued function of $\omega$ : any rational and integral function of $\phi \omega, \psi \omega, \chi \omega$ is termed a Modular Function. It is right to add that the definitions extend only to those values of $q$ for which the series are convergent, or (what is the same thing) $\omega$ regarded as a point must be situate within a certain region of the upper infinite half-plane $y=+$.

The requisite formulæ for the Theta-Functions are obtained from Jacobi's fundamental formula for the multiplication of four Theta-Functions; and the Elliptic Functions are introduced by means of their definitions in terms of the Theta-Functions: and the whole theory of Elliptic Functions is thus brought into connexion with the Theta and Omega Functions. The theory of Transformation depends in a great measure on the arithmetical and geometrical theory of binary matrices, of which the constituents are integral numbers; this theory plays an extensive part throughout the memoir.

An abstract of the contents of the memoir is as follows:

Arts. 1-14. Definitions and Elementary Properties of the Theta, Omega, and Elliptic Functions.

15-23. Arithmetical Theory of Binary Matrices.

24-34. The Transformation of the Theta and Omega Functions.

35-45. Geometrical Representation of Binary Quadratic Forms.

46-51. Geometrical Representation of the Modular Functions

$$
\Phi \omega \text { and } \Psi \omega\left(=\phi^{8} \omega, \psi^{8} \omega\right) .
$$

52-58. The Modular Equation.

59-62. The Equation of the Multiplier.

63-73. The Modular Curves.

74-82. Theory of the Modular Functions $\phi \omega$ and $\psi \omega$.

83-88. Theory of the Modular Function

$$
T \omega=\left(1-\chi^{24} \omega\right)^{3} \div \chi^{48} \omega
$$

89-90. The Differential Equation of the Modular Equations and Curves (this last section somewhat incomplete).

A good deal of the same ground is gone over in Weber's Elliptische Functionen und Algebraische Zahlen (8vo. Brunswick, 1891), a work which exhibits in a very compendious form the higher parts of the theory of Elliptic Functions, and which well deserves to be carefully studied. 